\title{
Bop encodes a muscle-restricted protein containing MYND and SET domains and is essential for cardiac differentiation and morphogenesis
}

\author{
Paul D. Gottlieb ${ }^{1 \star}$, Stephanie A. Pierce ${ }^{2,3 \star}$, Robert J. Sims III ${ }^{1 \star}$, Hiroyuki Yamagishi ${ }^{2,3}$, Elizabeth K. Weihe ${ }^{1}$, \\ June V. Harriss ${ }^{1}$, Shanna D. Maika ${ }^{1}$, William A. Kuziel ${ }^{1}$, Heather L. King ${ }^{1}$, Eric N. Olson ${ }^{3}$, Osamu Nakagawa ${ }^{2,3}$ \\ \& Deepak Srivastava ${ }^{2,3}$ \\ ${ }^{\star}$ These authors contributed equally to this work.
}

Published online: 1 April 2002, DOI: 10.1038/ng866

\begin{abstract}
Many transcription factors regulate specific temporal-spatial events during cardiac differentiation; however, the mechanisms that regulate such events are largely unknown. Using a modified subtractive hybridization method to identify specific genes that influence early cardiac development, we found that Bop is expressed specifically in cardiac and skeletal muscle precursors before differentiation of these lineages. Bop encodes a protein containing MYND and SET domains, which have been shown to regulate transcription by mediating distinct chromatin modifications. We show that $\mathrm{m}$-Bop is a histone deacetylase-dependent transcriptional repressor. Targeted deletion of Bop in mice disrupted maturation of ventricular cardiomyocytes and interfered with formation of the right ventricle. Normal expression of Hand2, a transcription factor essential for right ventricular development, in cardiomyocyte precursors is dependent upon $\mathrm{m}$-Bop. These results indicate that $\mathrm{m}$-Bop is essential for cardiomyocyte differentiation and cardiac morphogenesis.
\end{abstract}

\section{Introduction}

An understanding of early cardiogenesis is necessary for interventions in heart disease, a leading cause of death in adults and children. Transcriptional regulation of cardiogenesis has been explored in considerable detail, but the mechanisms controlling early steps of cardiomyocyte differentiation and maturation are largely unknown ${ }^{1-2}$. Cardiogenic precursors are specified in an anterior mesoderm field during early embryogenesis in response to secreted signals from the adjacent endoderm. Subsequently, a monolayer of cardiomyocytes produces a dense extracellular matrix (ECM) necessary for reciprocal signaling between the myocardial and endocardial layers of the developing heart tube ${ }^{3}$. Further differentiation of cardiomyocytes results in degradation of the ECM and invasion of more differentiated and less proliferative trabecular myocytes into the ECM. As this occurs, the linear heart tube forms a loop that begins to distinguish individual cardiac chambers and results in proper alignment of the inflow and outflow tracts of the heart.

As cardiomyocytes mature, distinct transcriptional programs emerge that regulate specific cardiac chambers. The discovery of distinct cis-acting elements governing gene expression in specific chambers first suggested the presence of such programs ${ }^{4}$. Evidence of chamber-specific trans-acting factors was revealed by the absence of a right ventricular segment in mice lacking the basic helix-loop-helix (bHLH) transcription factor, Hand2 (ref. 5), and by the left ventricle-specific expression of the closely related factor Hand1 (refs 5,6). Many chamber-specific transcription factors have subsequently been described ${ }^{7-9}$. The evidence of segmental regulation of cardiac transcription provides a mechanistic context for examining congenital heart defects in humans, which typically affect only a single chamber or region of the heart.

The recent discovery of proteins involved in chromatin modifications has revealed an additional mechanism of transcriptional regulation. Covalent acetylation of specific lysine residues on histone tails by histone acetyltransferases (HATs) promotes chromatin relaxation and transcription, whereas deacetylation by histone deacetylases (HDACs) in a reverse reaction results in chromatin condensation and silencing of gene transcription ${ }^{10-12}$. Some chromatin remodeling proteins that share a highly conserved SET domain, originally described in the proteins su(var)-39, enhancer of zeste and trithorax, can methylate unacetylated lysine residues on histone tails, resulting in more permanent silencing of transcription ${ }^{13-14}$ or, in some cases, activation of transcription ${ }^{15}$. No cardiac-specific function for proteins containing SET domains, however, has been described.

${ }^{1}$ Section of Molecular Genetics and Microbiology and Institute for Cellular and Molecular Biology, University of Texas at Austin, Austin, Texas 78712, USA. Departments of ${ }^{2}$ Pediatrics (Cardiology) and ${ }^{3}$ Molecular Biology, University of Texas, Southwestern Medical Center, 6000 Harry Hines Boulevard, Dallas, Texas 75390-9148, USA. Correspondence should be addressed to P.D.G. (e-mail: gottlieb@uts.cc.utexas.edu) or D.S. (e-mail: deepak.sirvastava@utsouthwestern.edu). 
Here we describe the activity of $\mathrm{m}$-Bop, a protein that contains a SET domain and also a MYND domain, the latter reported to be involved in recruitment of HDACs and transcriptional repres$\operatorname{sion}^{16,17}$. We isolated $B o p^{18,19}$ through a new subtractive hybridization approach to identify early cardiac-specific genes in chick embryos. Bop was first discovered in cytotoxic T cells and adult heart and skeletal muscle ${ }^{18}$; here we show that Bop is expressed specifically in cardiac and skeletal muscle precursors and in cardiomyocytes throughout chick and mouse development, beginning before cardiac differentiation. m-Bop can interact with HDACs and can function as a transcriptional repressor. Targeted deletion of Bop in mice revealed its essential role in ventricular cardiomyocyte maturation and right ventricular development. Hand2 expression in cardiac precursors was dependent upon Bop, revealing a likely mechanism, in part, for hypoplasia of the right ventricle and ventricular maturation defects in Bop mutants. These results indicate that the cardiac-restricted $\mathrm{m}$ Bop, containing MYND and SET domains, is essential in cardiomyocyte differentiation and chamber-specific development.

\section{Results}

Isolation of chicken m-Bop by subtractive hybridization In chick embryos, bilaterally symmetric cardiac precursors become specified by Hamburger and Hamilton stage 5 (ref. 20) and express the early cardiac-specific gene, $N k x 2-5$ (refs 21,22). To find genes that might be essential for the early period of cardiac specification and differentiation, we used a subtractive hybridization approach to isolate genes expressed specifically in the cardiac precursors at Hamburger and Hamilton stage 5. To avoid cloning genes expressed in the nearby neural field or abundant sarcomeric transcripts, we developed a modification of PCR-based subtractive hybridization that allowed enrichment of transcripts common to two RNA populations but absent in a third RNA pool. Using this new method, which we termed subtractive and selective PCR amplification (SSPA), we attempted to isolate genes expressed in both the presumptive heart field (stage 5) and in the straight heart tube (stage 10), but not in the posterior, non-cardiogenic region of the embryo. Ninety-nine percent of cDNA clones isolated by this modified method were anchored by unique adaptors, indicating that genes expressed in both desired
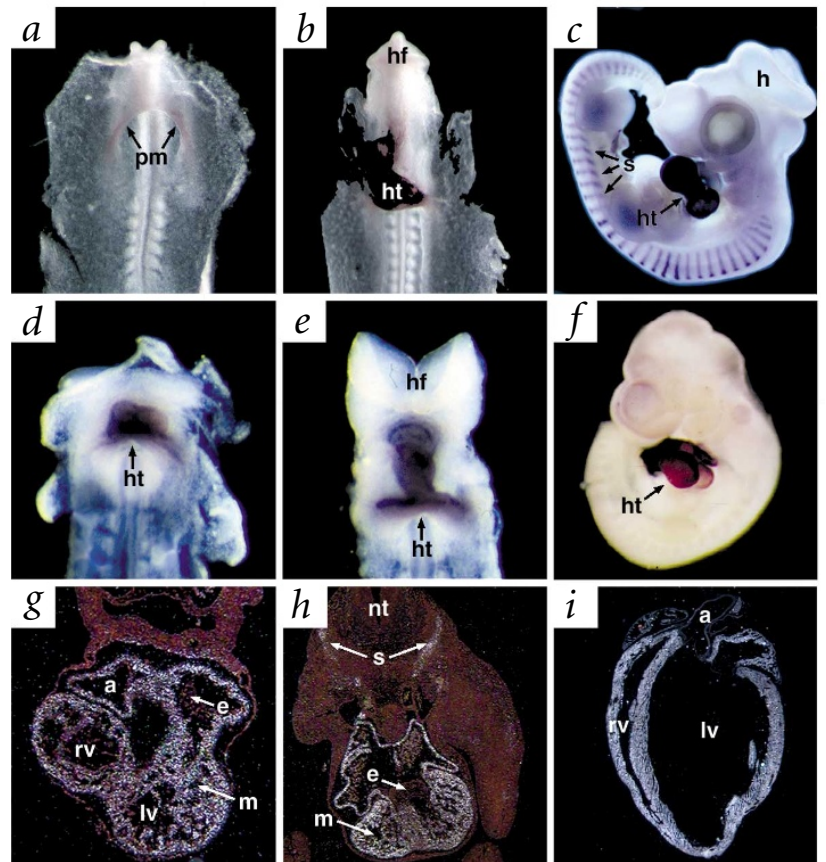

tissues were isolated. This modification allowed for efficient isolation of many non-sarcomeric, early cardiac-specific genes that will be described elsewhere (O. Nakagawa, E.N. Olson \& D. Srivastava, manuscript in preparation).

One of the sequences isolated in this screen showed homology to Bop, which has previously been described as being expressed in cytotoxic T cells ( $\mathrm{t}$-Bop), adult heart and adult skeletal muscle (skm-Bop, referred to here as $\mathrm{m}$-Bop $)^{18,19}$. Low-stringency screening of an embryonic chick library for the full-length cDNA revealed the presence of two closely related species, each of which shared $90 \%$ amino-acid similarity with mouse m-Bopl or mouse $\mathrm{m}$-Bop2, respectively. Based on the similarity with its mouse orthologs, we conclude that these cDNAs represent chick mBop1 and chick m-Bop2. m-Bop1 and m-Bop2 are encoded by the same gene $(B o p)^{19}$ and represent isoforms that differ only by an insertion of 13 amino acids that is due to alternative splicing. Because of the similarity between the two, we will refer to both isoforms collectively as m-Bop.

\section{Bop expression and structure}

To examine the expression of Bop during embryogenesis, we carried out whole-mount and section in situ hybridization. In chick, Bop transcripts were detectable by RT-PCR in the heart field at stage 5 (data not shown) and by in situ hybridization at stage 7 , before the onset of cardiomyocyte differentiation (Fig. 1a). Cardiac expression of Bop continued in the straight heart tube (stage 10) and throughout cardiogenesis (Fig. 1b,c). Expression in the myotome of the somites, which later contributes to skeletal muscle, was also abundant (Fig. 1c). Similar studies of mouse Bop during embryogenesis revealed conservation of cardiac-specific gene expression as early as embryonic day (E) 7.75, continuing throughout development (Fig. $1 d-f$ ). By radioactive section in situ hybridization, mouse Bop transcripts were localized to the myocardial, but not endocardial, layer of the developing heart and persisted in post-natal cardiomyocytes (Fig. $1 g-i$ ). Expression of Bop was uniform in all chambers of the heart and was also detected in the myotome of the somites (Fig. 1h).

The m-Bop 2 isoform encoded by Bop is a protein of 472 aa that contains a MYND domain, with its two predicted zinc fingers, and a SET domain, both of which have been implicated in chromatin remodeling (Fig. 2a). The SET domain of m-Bop is split into two segments (a S-ET domain ${ }^{15}$ ) separated by the MYND domain and other sequences. The MYND domain and the 'ET' portion of the S-ET domain are also present in a Bop isoform specific to thymus and cytotoxic T cells (t-Bop) ${ }^{19}$. The MYND domain of $\mathrm{m}$-Bop is most similar to that found in the ETO protein (also called CBFA2T1), which is translocated in human leukemias $(\mathrm{t}(8 ; 21))$, and is involved in recruitment of HDACs and transcriptional silencing (Fig. 2b) ${ }^{16-17}$. SUV39H1, which contains a conserved SET domain, possesses histone methyl transferase (HMT) activity that is dependent upon several conserved amino-acid residues within the SET domain ${ }^{13}$. m-Bop shares most of these essential residues, all within the 'ET' region, as well as the carboxy-terminal cysteine-rich region, with SUV39H1 (Fig. 2c).

Fig. 1 Cardiac- and somite-specific expression of Bop in chick and mouse. $a, b$, Whole-mount in situ hybridization revealed Bop expression in chick, beginning at Hamburger and Hamilton stage 7 in the precardiac mesoderm (pm; a), followed by heart (ht)-restricted expression at stage 10 (b). c, By stage 20 in chick, expression of Bop is seen in the heart and somites (s). $d-f$, Heart-specific expression of Bop in mouse at E7.75 (d), E8.0 (e) and E10.5 ( $f$ ), as seen by whole-mount in situ hybridization. $\boldsymbol{g}, \boldsymbol{h}$, Transverse section in situ analysis revealed expression of mouse Bop specifically in the myocardium $(\mathrm{m})$ of the heart at E9.5 $(\mathrm{g})$, as well as in the somites at E12.5 $(h)$. No expression of Bop is seen in the endocardium $(e ; g, h)$ $i$, Section in situ hybridization revealed Bop expression in the adult mouse heart. a, atria; hf, head-fold; $h$, head; Iv, left ventricle; $n t$, neural tube; rv, right ventricle. 
m-Bop can function as a transcriptional repressor

Because MYND and SET domains can promote chromatin remodeling and transcriptional repression, we examined the subcellular localization of $\mathrm{m}$-Bop2 to determine whether it could function as a transcriptional regulator. Immunocytochemistry using a hamster anti-mouse $\mathrm{m}$-Bop monoclonal antibody generated against the $\mathrm{C}$-terminal half of the protein shared by all $\mathrm{m}$ Bop isoforms ${ }^{19}$ revealed Bop expression in both cytoplasm and nucleus of undifferentiatied myoblasts (Fig. $2 d$ ).

To test for transcriptional repressor activity, we used a system in which a luciferase reporter gene was activated by a fusion protein of the LexA DNA-binding domain fused to the potent activation domain of the viral coactivator protein,VP16 (LexA-VP16) $^{23}$. Interaction of GAL4-binding sites, located between the LexA-binding sites and the reporter, with a GAL4m-Bop2 fusion protein consistently inhibited luciferase expression in a dosedependent manner, compared with LexA-VP16 alone or both LexA-VP16 and GAL4-DBD (Fig. 2e). This effect is probably not due to steric hindrance, as other GAL4-fusion proteins did not show similar repression of LexA-VP16 (data not shown). Using the reverse constructs, a LexA-m-Bop2 fusion protein suppressed transcriptional activation by GAL4-VP16 in a similar manner (data not shown).

In a second assay for the effect of $\mathrm{m}$ Bop2 on transcriptional activity, we used a luciferase reporter gene under the influence of a minimal SV40 promoter. We used a series of SV40-luciferase reporters that contained zero or five copies of the GAL4-UAS upstream of the SV40 promoter at various distances (Fig. $2 f$ ). In transient transfections of $10 \mathrm{~T} 1 / 2$ cells, GAL4-m-Bop2 consistently inhibited transcription in a dose-dependent manner, regardless of the distance between

Fig. $2 \mathrm{~m}$-Bop is an HDAC-dependent transcriptional repressor. $a$, Predicted protein structure of $\mathrm{m}$-Bop2, with positions of MYND and S-ET domains indicated. The conserved cysteine-rich region is indicated in red. $\boldsymbol{b}$, Comparison of the MYND zinc-finger domain of mouse m-Bop with other MYND domain-containing proteins. c, Comparison of the m-Bop2 S-ET domain with other SET domain-containing factors. Conserved residues are indicated by black shading, similar residues are indicated by gray and residues necessary for HMT activity are indicated by purple or red. An asterisk marks a prominent difference between $\mathrm{m}$-Bop2 and SUV39H1 at a residue that affects HMT activity $^{13}$. The location of the MYND domain is indicated by an open arrowhead. Mm, Mus musculus; Hs, Homo sapien; Ds, Drosophila. d, Immunocytochemistry of C2C12 cells with Bop monoclonal antibodies. Nuclear or cytosolic regions are indicated by DAPI or tubulin staining, respectively. e, C3H/10T1/2 cells were transiently transfected with the pL8G5-luc reporter along with the indicated LexA or GAL4 mammalian expression vectors. GAL4-m-Bop2 was co-transfected with LexA-VP16 in increasing amounts ( $1 \mathrm{X}=0.3 \mu \mathrm{g})$. Relative luciferase activity was normalized for transfection efficiency by co-transfection with GFP and FACS analysis. $f$, 5XGAL4-SV40-luciferase reporter co-transfected with or without GAL4-m-Bop2. Distances of 5XGAL4 sites from SV40 promoter are shown. $g$, 10T1/2 cells were transfected with GAL4-DBD or GAL4-m-Bop2 along with 5XGAL4-SV40-luc $(1 \mathrm{X}=1 \mu \mathrm{g})$ as indicated. TSA $(150 \mu \mathrm{M})$ was added $24 \mathrm{~h}$ after transfection. Results in e- $g$ are shown as the mean percentage and standard error of normalized activation from three separate experiments. $\boldsymbol{h}$, 293T cells were transfected with FLAG-tagged HDAC1, -2 or -3 along with GAL4-DBD or GAL4-m-Bop2. Proteins from cell lysates were immunoprecipitated with a Bop-specific monoclonal antibody, separated by SDS-PAGE and immunoblotted with a FLAG-specific antibody. Input is shown in the lower panel. the GAL4 binding sites and the SV40 promoter. This suggests that $\mathrm{m}$-Bop2 represses transcription by an active mechanism, rather than by simply interfering with the transcriptional thery. The $\mathrm{m}$-Bopl isoform repressed transcription in a manner similar to $\mathrm{m}$-Bop2 (data not shown). These findings sug-

\section{Transcriptional repression by $\mathrm{m}$-Bop requires HDAC} activity

m-Bop has repressor activity and contains a MYND domain $i^{24}$, we used the potent HDAC inhibitor trichostatin A (TSA) ${ }^{25}$ to test whether m-Bop2 might repress transcription by recruiting HDACs. Addition of $150 \mu \mathrm{M}$ TSA significantly abrogated GAL4-m-Bop2 repression of SV40 promoter-driven luciferase
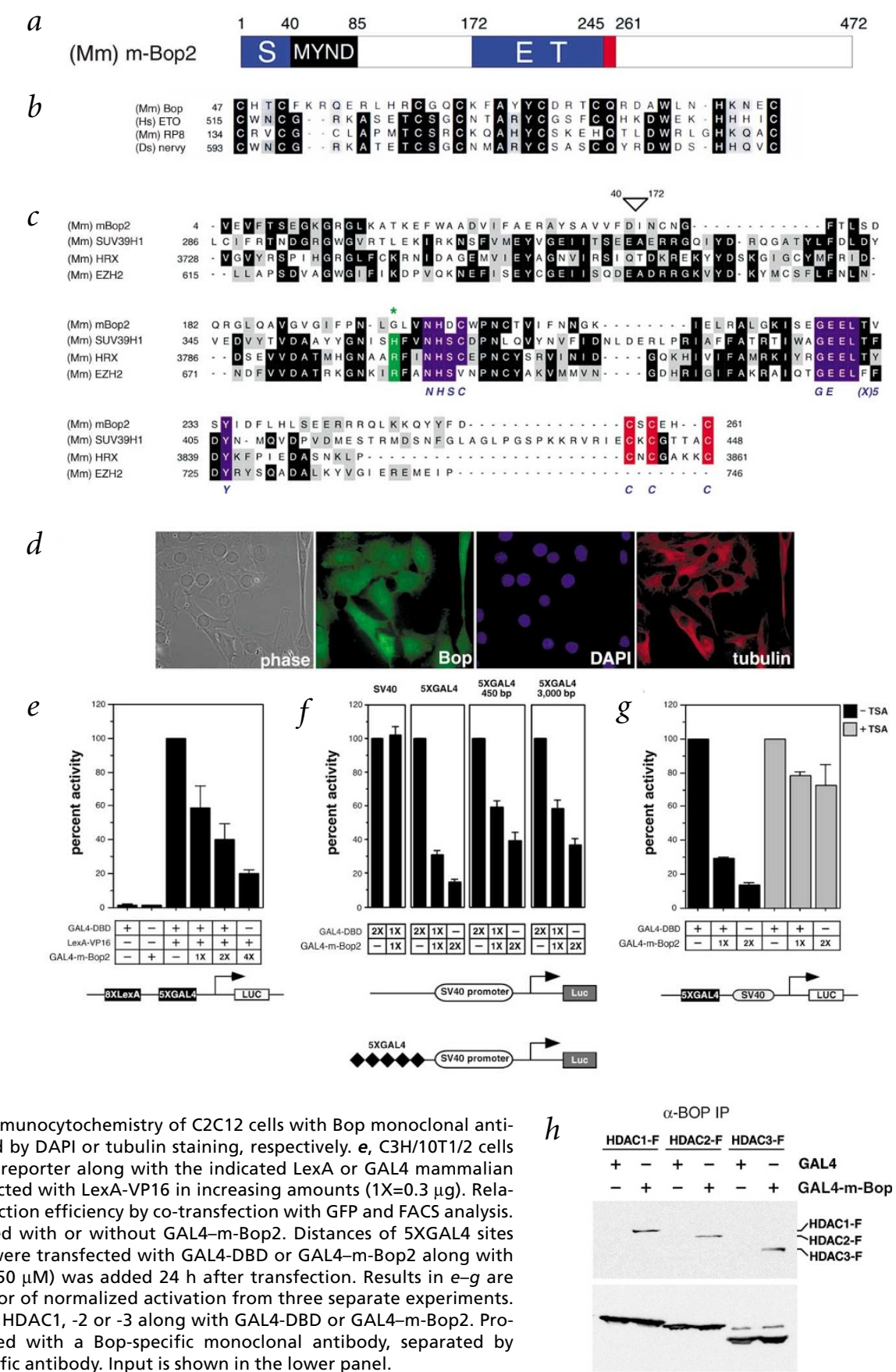
$a$

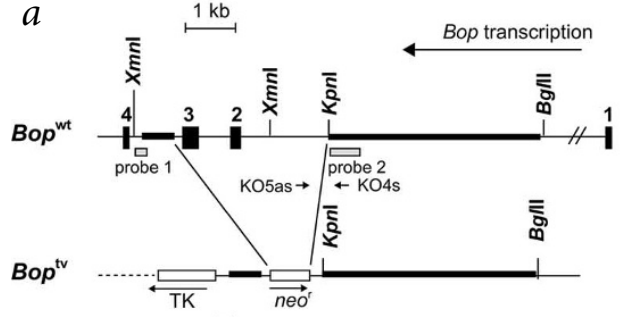

Bop null

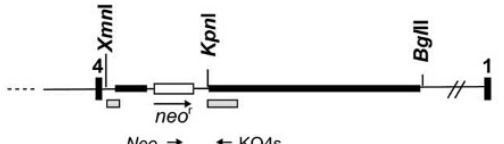
$\mathrm{NeO} \rightarrow \leftarrow \mathrm{KO} 4 \mathrm{~s}$

$b$
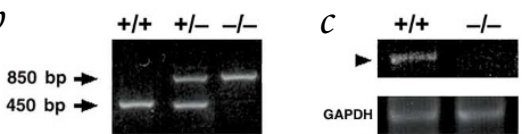
recruitment of HDAC activity (Fig. $2 g$ ).
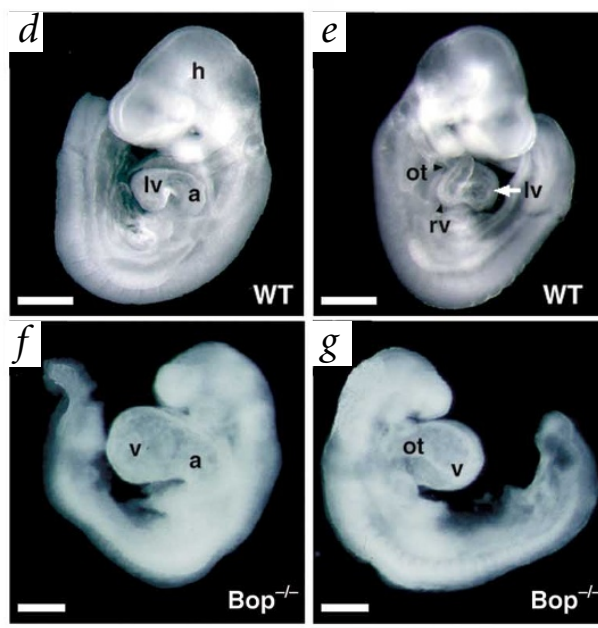

Fig. 3 Targeted deletion of Bop results in embryonic lethality, owing to cardiac enlargement. a, Organization of the Bop wildtype (Bop ${ }^{\mathrm{wt}}$ ) allele and targeting vector $\left(B o p^{\mathrm{t} v}\right)$. Homologous recombination resulted in replacement of exons 2 and 3 of Bop by neor. as shown in the Bop-null allele (Bop null). b, Genotyping of the wildtype $(+/+)$, heterozygous $(+/-)$ and homozygous (-I-) null loci for Bop.| c, RT-PCR for Bop transcripts in wildtype or Bop-null hearts. $\boldsymbol{d}-\boldsymbol{g}$, Left and right lateral views of wildtype (WT; $d, e)$ and Bop mutant $(f, g)$ embryos at E9.5 are shown. Gross analysis of mutant embryos shows growth retardation, an enlarged ventricular chamber (v) and a single ventricular segment, in contrast to wildtype embryos. Scale bars, $400 \mu \mathrm{M}$. a, atria; $h$, head; Iv left ventricle; ot, outflow tract; $r v$, right ventricle. expression in 10T1/2 cells, suggesting that the effect of m-Bop2 on expression of the reporter gene is mediated, at least in part, by

To understand the mechanism of TSA-mediated inhibition of $\mathrm{m}$ Bop2 activity, we tested the ability of m-Bop2 and HDAC to physically associate in vivo. We generated extracts of 293T cells transiently transfected with plasmids expressing GAL4-m-Bop2 or GAL4-DBD together with FLAG epitope-tagged HDACs (HDAC1, HDAC2 or HDAC3). Immunoprecipitation of cell lysates with a Bop-specific monoclonal antibody, followed by western blot with anti-FLAG antibody, demonstrated that all three class I HDACs coprecipitated with GAL4-m-Bop2 but not Gal4-DBD (Fig. $2 h$ ). In addition, anti-BOP mAb co-precipitated FLAG-tagged class II HDACs, HDAC4 and HDAC5, from cells co-transfected with GAL4-m-Bop2 but not GAL4-DBD (data not shown). Conversely, anti-FLAG antibody co-precipitated GAL4-m-Bop2 from lysates of 293T cells overexpressing GAL4-m-Bop2 and FLAG-HDAC1, but not GAL4-m-Bop2 alone (data not shown). Taken together, these results suggest that $\mathrm{m}$-Bop2 suppresses transcription, at least in part, by recruitment of HDAC activity.

\section{Bop $^{-/-}$mice show ventricular hypoplasia and expanded extracellular matrix}

The tissue and developmental expression pattern of Bop and its role as a transcriptional regulator prompted us to investigate its in vivo function using mice lacking functional Bop. We inactivated $B o p$ by replacing exons 2 and 3, which encompass the MYND domain, with the neomycin resistance gene $\left(n e o^{\mathrm{r}}\right)$ through homologous recombination (Fig. $3 a$; see Methods). Mice heterozygous with respect to the mutated Bop allele seemed normal and fertile. Genotyping of 112 progeny of heterozygous intercrosses, however, indicated that 71 (63\%) were heterozygous and $41(37 \%)$ contained only wildtype alleles (Fig $3 b)$. We found no evidence of stillborn pups, suggesting that embryos lacking a functional Bop allele died in utero.

We sacrificed females from timed heterozygous matings to determine the day of embryonic death. Embryos homozygous with respect to the mutated $B o p$ allele showed growth retardation by E9.5 and were dead by E10.5 (Fig. $3 d-g$ ). No Bop mRNA transcripts were detectable by RT-PCR from Bop mutant hearts at E9.5, indicating the absence of any residual spliced message (Fig. 3c). Gross examination of Bop mutant embryos at E9.5 revealed an enlarged heart with a more severe effect evident in the ventricle than in the atrium (Fig. 3f). In addition, there appeared to be a single leftside ventricular chamber (Fig. $3 g$ ).
Histologic analysis of Bop mutant hearts by serial transverse section along the anterior-posterior axis revealed that, although the hearts were enlarged compared with the wild type, the lumen of the heart was not dilated (Fig. $4 a-f$ ). Rather, the enlargement was secondary to a tremendous expansion of ECM, known as cardiac jelly, between the myocardial and endocardial layers of the developing heart (Fig. $4 b, d, f$ ). In addition, at no level of section were two distinct ventricular chambers detectable, as in the wild type (Fig. 4c), and the single ventricular chamber was directly connected to the outflow tract (Fig. $4 d$ ). The atrium was relatively unaffected, with normal amounts of ECM (Fig. 4f). Finally, formation of trabeculae, the finger-like myocardial projections indicative of cardiomyocyte differentiation and maturation, was limited in Bop mutants.

To understand the mechanism underlying the expansion of ECM in Bop mutants, we examined the composition and nature of ECM in hearts lacking Bop. As detected by electron microscopy and antibodies to numerous components of normal ECM, including fibronectin, collagen and hyaluronan, the ECM in mutant hearts seemed relatively normal in composition, but expanded compared with the wild type (Fig. $4 g, h$ and data not shown), although effects of hydration may also contribute. Because the enzyme hyaluronic acid synthase 2 (Has2) is necessary for ECM production ${ }^{26}$, we hypothesized that this gene might be upregulated in the absence of Bop. However, section in situ hybridization revealed no change in the level of Has 2 transcripts in Bop mutant hearts (Fig. 4i,j). Together, these data suggest that Bop may not directly regulate genes involved in production or degradation of ECM, but may affect a more general process reflected by the expansion of ECM.

\section{Cardiomyocyte maturation defect in $\mathrm{Bop}^{-/-}$mice}

Because cardiomyocytes undergo a critical maturation step between E8.0 and E9.5 that is manifested by a transition from production to degradation of $\mathrm{ECM}^{27}$, we assessed the degree of cardiomyocyte maturation by using several markers of cardiomyocyte differentiation in $B o p^{-1-}$ mice. Expression of markers of early cardiomyocyte differentiation, including myosin light chain, phosphorylatable, cardiac ventricles $(M y l p c)^{28}$ and myosin regulatory light chain A $(M y l c 2 a)^{29}$, which are expressed before cardiomyocyte contractions, were normal in Bop-null embryos (Fig. 5a,b and data not shown). Hand1, a left ventricular marker, was expressed normally in Bop mutants and was detected throughout the ventricular chamber (Fig. $5 c, d$ ). In contrast, Hand2 (ref. 30), which is necessary for formation of 
the right ventricle, was not detected in the hearts of $B o p^{-1-}$ embryos at E9.0, although normal expression was observed in the pharyngeal arches and lateral mesoderm (Fig. $5 e, f$ ). We examined Hand 2 expression in $B o p^{-1-}$ mice at a slightly earlier time point than the wildtype embryos shown, in an attempt to capture potential Hand2 expression in a rudimentary right ventricle. To test whether this result simply reflected the absence of a right ventricle in the $B o p^{-1-}$ mice, we examined embryos at even earlier stages, before cardiac formation. In embryos at E7.75, Hand2 was selectively downregulated in the precardiac mesoderm (cardiac crescent) of $B o p^{-1-}$ mice (Fig. $5 g, h$ ), but a small amount of expression was still detectable. Expression of Hand2 was intact bilaterally in the lateral mesoderm, providing an internal control for RNA integrity in the $B o p^{-1-}$ embryo. This result indicates that $\mathrm{m}$-Bop regulates expression of Hand 2 in precardiac mesoderm well before organogenesis or any visible phenotype in the Bop mutant.

We examined alteration of Hand2 expression in the heart by radioactive-section in situ hybridization, which revealed lower amounts of Hand2 expression in cardiomyocytes of $B o p^{-1-}$ mice than in the wild type (Fig. 5i,j). Expression of the transcription factor Nkx2-5 in tissue sections of $B o p^{-/-}$mice was normal, providing a control for intact gene expression in the $B o p^{-1-}$ mice heart (Fig. $5 k, l)$. To further assess the effects of Hand 2 downregulation in $B o p^{--}$mice, we considered whether expression of any Hand2-dependent genes might be altered in the absence of Bop. Irx4, a ventricular-specific homeobox gene, was recently shown to be partially downregulated in mice lacking Hand2 (ref. 31). In the $B o p^{-1-}$ mice ventricle, Ir $x 4$ transcripts were detectable but downregulated, compared with wildtype littermates, consistent with the downregulation of Hand2 (Fig. $5 m, n$ ). Expression of other transcription factors was equal in wildtype and $B o p^{-1-}$ mice on serial sections (Fig. $5 k, l, o, p$ ), suggesting that alteration in RNA integrity was not the cause of decreased Irx4 expression.

Although whole-mount in situ hybridization suggested that the predominant portion of the residual ventricle was molecularly a left ventricle, we investigated this with higher resolution using section in situ hybridization. Tbx5, which is normally expressed at highest levels in the atria and left ventricle ${ }^{32}$, was expressed at comparable levels in the $B o p^{-1-}$ and wildtype heart (Fig. 5o,p), consistent with the conclusion that the single ventricle in the absence of Bop is, on a molecular basis, primarily a left ventricle. However, a

Fig. 4 Single-ventricle and extracellular matrix expansion in $\mathrm{Bop}^{-1-}$ embryos. $a-f$, Transverse hematoxylin and eosin-stained sections of wildtype and $\mathrm{Bop}^{-1-}$ embryos at the level of the outflow tract (ot; $a, b)$ or ventricular chambers $(v ; c, d)$, show reduced trabeculae, an enlarged single ventricular chamber filled with extracellular matrix $(*)$ between the endocardial $(e)$ and myocardial $(\mathrm{m})$ cell layers $(b, d, f)$ and a nondistinct right ventricle ( $r v ; d)$, compared with wildtype embryos (c). Note the small lumen inside the endocardial layer $(b, d)$. Transverse section at the level of the atria shows a normal-sized atrial chamber (a) in the Bop ${ }^{-1-}$ embryo $(f)$, as compared with the wildtype embryo (e). Scale bars represent $100 \mu \mathrm{M}$. g, $\boldsymbol{h}$, Histochemistry using a biotinylated hyaluronan-binding protein shows levels of hyaluronan (*) in ECM of Bop mutant embryos at E9.5 $(h)$ similar to those of wildtype embryos $(g)$. Wildtype embryo at E8.5 is shown because minimal ECM is present in wildtype hearts at E9.5. Hyaluronan expression in cardiomyocytes is also similar in both. $i, j$, In situ hybridization of a transverse section of a Bop-null embryo shows expression of hyaluronic acid synthase 2 (Has2; $j$ ) at amounts similar to that of the wild type (i). $\boldsymbol{k}_{\boldsymbol{l}} \boldsymbol{l}$, Bright field views of $i$ and $j$, respectively; the arrowhead indicates the atrioventricular canal. Iv, left ventricle; nt, neural tube.

small region of cells leading into the outflow tract of the $B o p^{-/-}$ heart showed lower levels of $T b x 5$ expression (Fig. $5 p$ ), similar to the lower expression level in wildtype right ventricle (Fig. 5o), suggesting that these cells may represent remnants of the right ventricle. To distinguish if this subset of cells represents outflow tract or right ventricle, we examined Hand1 expression, which marks only left ventricle and outflow tract (Fig. 5q). The cells with lower $T b \times 5$ expression did not have detectable Hand 1 transcripts (Fig. 5r), indicating that a rudimentary, hypoplastic right ventricle was present. Not surprisingly, $5 \pm 1$ of 40 cells in the anterior region of the $B o p^{-1-}$ looping heart tube at E9.0 were undergoing apoptosis, as seen by TUNEL assay, compared with 0 of 80 cells in a comparable region in wildtype hearts $(n=4$, $P<0.05)$. Whereas the hypoplasia of a right ventricular segment, similar to Bop mutants, has been well documented in Hand2 mutants ${ }^{33}$, the space between endocardium and myocardium is not as prominent as in $B o p^{-/-}$mice (Fig. 5t,u). However, this space was greater in Hand2--- hearts at E9.5 than in wildtype littermates (Fig. $5 s, t$ ) and was in sharp contrast to the minimal ECM detected in mice lacking $N k x 2-5$ (Fig. $5 v$ ) or Mef2c (data not shown) at E9.5.

\section{Discussion}

We have cloned chick Bop, using a new screen to search for early cardiac-specific genes, and show that Bop has a crucial role in mouse cardiogenesis. Bop is expressed early in development, being detected in the anterior half of chick embryos at
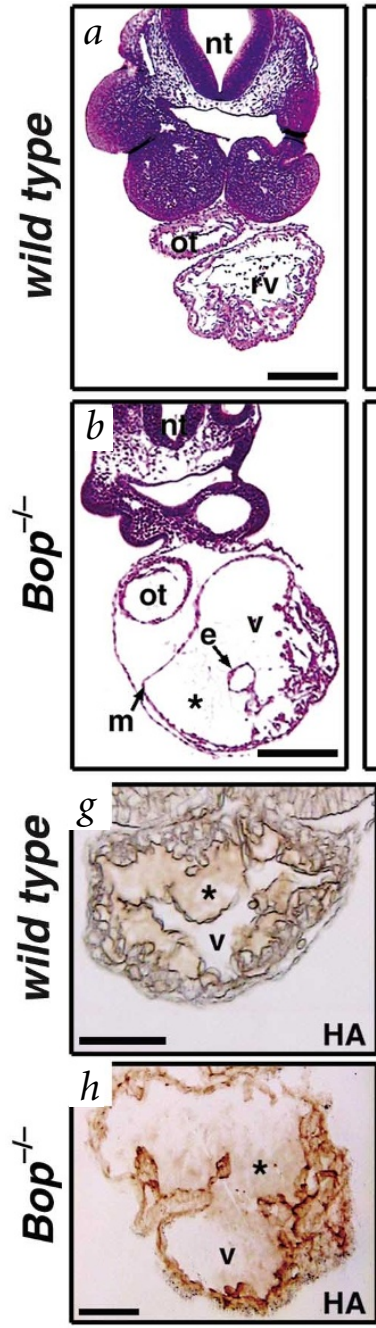
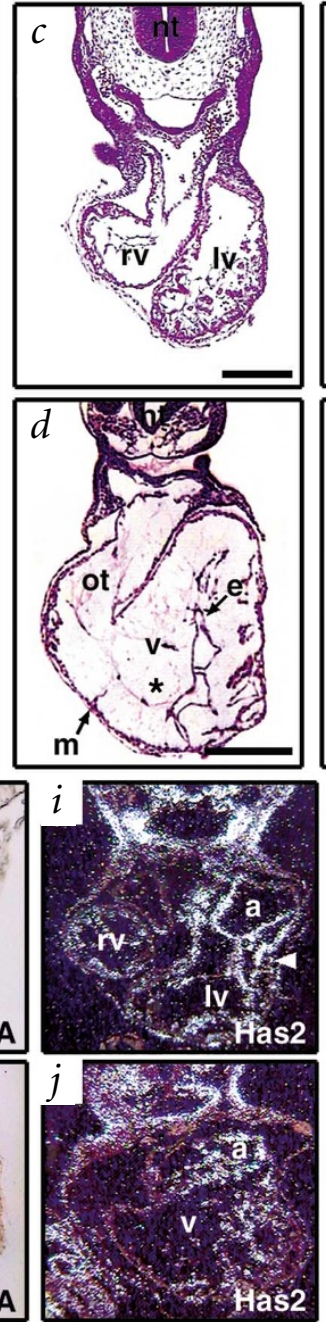
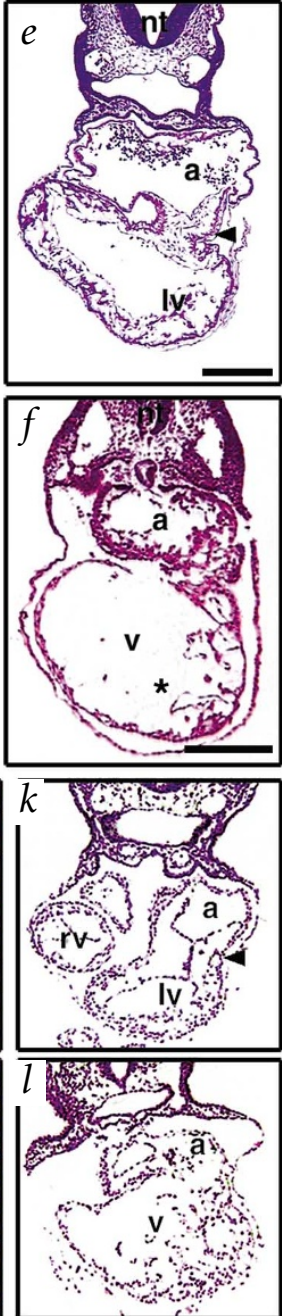
Hamilton and Hamburger stage 5 and in the precardiac mesoderm (cardiac crescent) of mouse embryos at E7.75. m-Bop is the first cardiac and muscle-specific protein containing MYND or SET domains to be identified and can function as an HDAC-dependent transcriptional repressor. Targeted deletion of Bop in mice indicates that it is required for normal expression of Hand2 in the precardiac, but not lateral, mesoderm and that Bop is necessary for maturation of cardiomyocytes and morphogenesis of the right ventricle.

Selective and subtractive PCR amplification. Classical subtractive hybridization is an efficient method for identifying differences in the transcriptional profile between tissues; however, it is limited by its inability to compare transcripts in more than two RNA pools. We have devised an efficient method, SSPA, to compare RNA species present in two populations but absent from a third. We have applied this method to clone early cardiac-specific genes; SSPA will probably be useful for profiling other dynamic developmental events.
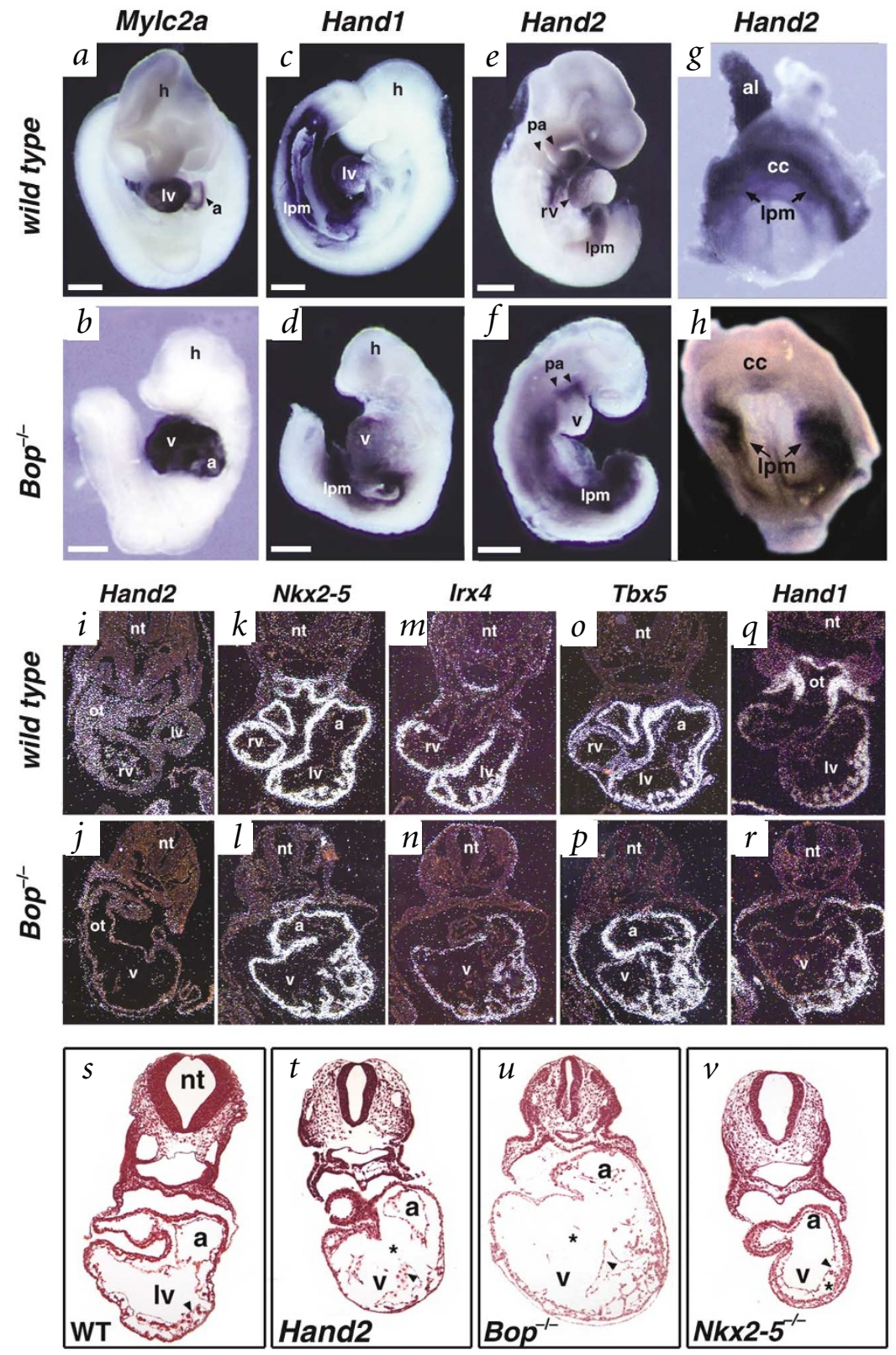

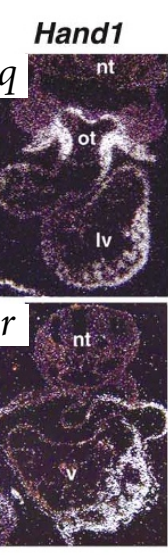

Cardiomyocyte maturation defects in $B o p^{-1-}$ mice. The paucity of trabeculations, expansion of ECM and downregulation of Hand2 in $\mathrm{Bop}^{-/-}$cardiomyocytes are consistent with a disruption of differentiation into a more mature cardiomyocyte. Although there are no specific molecular markers for the maturation of cardiomyocytes between the straight heart tube and the looped heart tube stages, there are clear morphologic changes that normally occur. The early heart tube is mostly composed of an ECM that is secreted by a monolayer of myocardial cells and later degraded predominantly by more differentiated cardiomyocytes ${ }^{27}$. The degree of expansion of ECM observed in $B o p^{-l-}$ mice exceeds that seen in any previously described mouse mutant and may be secondary to an arrest of the transition from a partially differentiated to a more mature cardiomyocyte. Alternatively, it is possible that m-Bop directly affects gene transcription of a unique set of genes involved in the production or degradation of ECM, including proteases or inhibitors of proteases. However, in light of the absence of detectable differences in the composition of ECM or synthetic enzymes in $B o p^{-/-}$mice and the presence of Hand2 downregulation, we currently favor the more general interpretation involving a role for $\mathrm{m}$-Bop in a critical step in cardiomyocyte differentiation, manifested as improper management of ECM.

Fig. 5 Hand2 is downregulated in cardiomyocyte precursors of Bop ${ }^{-1-}$ embryos. a- $h$, Whole-mount in situ hybridization of wildtype and $\mathrm{Bop}^{-1-}$ embryos. $a, b$, Expression of Mylc2a is unchanged in $B o p^{-1}$ mice, as compared with the wild type. $c-f$, Hand 1 is expressed normally in the single ventricle (v) and lateral-plate mesoderm (Ipm) of the $B o p^{-1-}$ mice embryo (d), compared with the wild type (c), whereas Hand2 shows decreased expression in the ventricle of a $\mathrm{Bop}^{-1-}$ mice at E9.0 (f), compared with the wild type at E9.5 (e); expression in the lateral-plate mesoderm (Ipm) and pharyngeal arches (pa) was intact. Scale bars represent $400 \mu \mathrm{M}$. Bop ${ }^{-1-}$ embryos were examined at E9.0 to search for expression in a rudimentary right ventricle and were also exposed longer in an attempt to reveal low levels of Bop expression in the heart ( $f$ ). $\boldsymbol{g}, \boldsymbol{h}$, Expression of Hand2 is lower in the cardiac crescent (precardiac mesoderm) of Bop $^{-1-}$ embryos at E7.75 (h) than in wildtype embryos $(g)$, but is unchanged in the bilateral Ipm. $i, j$, Transverse-section in situ hybridization of E9.0 embryos shows decreased expression of $H a n d 2$ in the ventricular chamber $(v)$ and outflow tract (ot) of the $\mathrm{Bop}^{-1-}$ embryo (j), compared with the wildtype embryo, at the level of the connection between the right ventricle and the outflow tract (i). $k, l$, Expression of $N k \times 2-5$ in the ventricle (v) and atria (a) of the $B o p^{-1-}$ embryo (l), is normal compared with wildtype expression $(k)$, as seen by section in situ hybridization. $m, n$, Expression of Irx4 is lower in the single ventricular chamber of the $B_{0} p^{-1-}$ embryo $(n)$ than in the wild type $(m)$. o, p, Tbx5 is expressed in the single $B o p^{-1-}$ ventricle $(p)$ at levels comparable to those of the wild type (o). A small group of cells on the right side had lower levels of expression $(p)$, similar to the right ventricle of the wild type (o). $q, r$, Serial section of Hand1 expression is notable for lacking expression in this subset of cells but having robust expression in the remainder of the ventricle $(r)$. The wild type shows specific expression in the left ventricle and outflow tract $(q)$; the full right ventricle is not shown in this section. $s-v$, Transverse sections of wildtype (s), Hand2 ${ }^{-t-}(t)$, $\mathrm{Bop}^{-1-}(u)$ and $\mathrm{Nk}^{-2-5^{-1-}}(\mathrm{v})$ embryos at E9.5 are shown at the level of the atrio-ventricular junction. Arrowheads are in the ventricular lumen and point to the endocardium. Images in $s-v$ are at equal magnification. Asterisks $(*)$ indicate the area of the ECM. al, allantois; cc, cardiac crescent; Iv, left ventricle; $\mathrm{nt}$, neural tube; rv, right ventricle. 
Chamber-specific functions of Bop. Although Bop is expressed throughout the chambers of the heart, its function during mouse cardiogenesis is relatively specific to the ventricular chambers. It is remarkable that the Bop mutant atrial chamber had no expansion of ECM. Within the ventricles, the cardiomyocytes of the right ventricle seemed to be more severely affected, but the residual myocytes of the left ventricle were also abnormal. This phenotype was similar to, although more severe than, the small right ventricle seen in Hand2 mutants ${ }^{5,33}$. While it is possible that hemodynamic alterations could contribute to the ultimate phenotype observed in any mouse model of cardiac defects, the similarities between Bop and Hand2 mutants and the early (E7.75) failure of Hand2 expression in the precardiac mesoderm in the absence of Bop suggest a more specific role for $B o p$ in ventricular development. A GATA-dependent enhancer upstream of Hand2 can direct cardiac gene expression of Hand2 in vivo ${ }^{34}$. Whether m-Bop directly or indirectly regulates Hand2 through this GATA site or through as-yet unknown sites has yet to be determined. It is more likely, however, that $\mathrm{m}$-Bop regulates an intermediate, as $\mathrm{m}$-Bop functions as a transcriptional repressor, although it is possible that $\mathrm{m}$-Bop could also function as an activator, given appropriate cofactors.

$\mathbf{m}$-Bop as a regulator of chromatin modification. The m-Bop protein contains a MYND domain homologous to that of the ETO protein, whose fusion with the AML1 protein in chronic myelogenous leukemia converts AML1, normally a transcriptional activator, into a transcriptional repressor ${ }^{24}$. The MYND domain of ETO is essential for this conversion and seems to function by recruiting the nuclear co-repressor, N-CoR, and the Sin3/HDAC complex to DNA sites specified by AML1 binding ${ }^{16,17}$. Similarly, we show that $\mathrm{m}$-Bop can function as a transcriptional repressor by interacting with HDACs. It will be interesting to determine whether the subcellular localization of $\mathrm{m}$-Bop is regulated or whether $\mathrm{m}$-Bop functions in a complex with other muscle-specific transcription factors that interact with HDAC, such as MEF2 factors ${ }^{35}$. Our preliminary data indicate that $\mathrm{m}$-Bop can interact with the musclespecific transcription factor skNAC (P.D.G. et al., manuscript in preparation), although the significance of this interaction has yet to be determined.

Proteins containing SET domains are also believed to regulate chromatin structure ${ }^{36}$. Although the mechanism through which most SET-domain proteins achieve this is unknown, the SET domain-containing human SUV39H1 and mouse Suv39h1 proteins have recently been shown to modulate higher-order chromatin structure and, thus, gene expression, by means of intrinsic HMT activity ${ }^{13,36}$. Whether the S-ET domain of m-Bop contains intrinsic HMT activity or serves some other function has yet to be determined. However, analysis of sequence databases reveals a number of genes in yeast, plants, Drosophila and mammals that contain S-ET domains with intervening sequences containing MYND domains, similar to Bop (R. Sims and P. Gottlieb, manuscript in preparation), suggesting a conserved function for this combination of domains.

Our findings indicate that Bop regulates a critical maturation step necessary for development of ventricular cardiomyocytes and formation of the right ventricle. To our knowledge, $\mathrm{m}$-Bop is the first cardiac and muscle-specific SET or MYND domain-containing protein identified and, as such, may be part of a muscle-specific module for regulation of higher-order chromatin structure.

\section{Methods}

Cloning of chicken m-Bop cDNA. Our modification of the PCR Select (Clontech) method of subtractive hybridization, SSPA, resulted in cloning of a 391-bp fragment of chick Bop from chick hearts at stages 5 and 10 . Briefly, we ligated cDNA pools from presumptive heart fields at stage 5, including all three germ layers, or straight heart tube at stage 10 with different oligonucleotide adaptors and separately hybridized them with excess cDNA from the non-cardiogenic posterior region of embryos at stage 5. We mixed and further hybridized the two samples to allow complementary single-stranded DNA to anneal. We preferentially amplified double-stranded DNA with complementary strands derived from both tester pools using primers designed to anneal to the unique adaptors ligated on the two tester populations. We screened a 64-74-h chick embryonic heart $\lambda$-cDNA library (Stratagene) using the 391-bp [ $\left.{ }^{32} \mathrm{P}\right]$-labeled chick Bop fragment cloned by SSPA, under conditions of low stringency in hybridization buffer consisting of $30 \%$ formamide, $6 \times$ SSPE, $0.5 \%$ SDS and $100 \mu \mathrm{g} \mathrm{ml}^{-1}$ denatured salmon sperm DNA at $42^{\circ} \mathrm{C}$ overnight, as previously described ${ }^{30}$. Filters were washed with $2 \times$ SSC, $0.1 \%$ SDS at $42{ }^{\circ} \mathrm{C}$ for $10 \mathrm{~min}$ followed by $0.1 \times \mathrm{SSC}, 0.1 \%$ SDS at $65^{\circ} \mathrm{C}$ for $15 \mathrm{~min}$. Positive clones were purified, excised into pBluescript plasmids, sequenced by automated sequencing and analyzed by BLAST search.

Whole-mount and section in situ hybridization. For whole-mount in situ hybridization, we prepared digoxygenin-labeled RNA probes by in vitro transcription. We isolated mouse or chick embryos at E7.5-E10.5 or stages 5-24, respectively, in PBS and removed the pericardium, followed by fixation in $4 \%$ paraformaldehyde/PBS at $4{ }^{\circ} \mathrm{C}$ overnight. We carried out whole-mount in situ hybridization as previously described ${ }^{37}$. For section in situ hybridization, we generated $\left.{ }^{35} \mathrm{~S}\right]$-labeled antisense riboprobes by in vitro transcription and carried out in situ hybridization on mutant and wildtype embryos at E9.0-E12.5 and adult heart, as previously described ${ }^{30}$.

Cell culture and transient transfections. We grew C3H 10T1/2, C2C12 and $293 \mathrm{~T}$ cells (ATCC) in DMEM (Life Technologies) supplemented with $10 \%$ fetal bovine serum at $37^{\circ} \mathrm{C}$ in an atmosphere of $5 \% \mathrm{CO}_{2}$ in air. We transfected 293T and 10T1/2 cells using FuGENE6 reagent (Boehringer Mannheim). For luciferase assays, we harvested cells $48 \mathrm{~h}$ after transfection. The total DNA concentration was held constant by adding the GAL4-DBD expression plasmid. The pL8G5-luciferase reporter contains eight LexA-binding sites upstream of five copies of the GAL4-UAS site. The pGL2-5XGAL4-SV40 luciferase reporter (J. Milbrandt) contains five copies of the GAL4-UAS upstream of the SV40 promoter. For TSA inhibition assays, we placed cells in media with or without $150 \mu \mathrm{M}$ TSA (Calbiochem) $24 \mathrm{~h}$ after transfection. For immunoprecipitation experiments, we transfected $2 \times 10^{6} 293$ T cells with FLAG-tagged HDAC1, -2 or -3 (S.L. Schreiber and E. Seto), GAL4-DBD or GAL4-m-Bop2 in various combinations. We used a total of $8 \mu \mathrm{g}$ of DNA and $24 \mu \mathrm{l}$ FuGENE6 in each transfection. Cells were lysed in RIPA buffer containing protease inhibitors $48 \mathrm{~h}$ after transfection.

Luciferase assays. We analyzed cell lysates with a Dynex microtiter luminometer. Relative light units obtained from the pL8G5-luciferase reporter (Luciferase Assay Kit, Promega) were normalized by transfection efficiency and protein concentration. We determined transfection efficiency by FACS (FACSCalibur, Becton Dickinson) analysis of cells co-transfected with the pEGFP-N2 expression plasmid (Clontech). Relative light units obtained from the pGL2-5XGAL4-SV40 reporter (Dual Luciferase Assay System, Promega) were normalized to a co-transfected pRL-TK expression plasmid. Percent activity was determined in relation to the GAL4-DBD. We carried out three independent experiments to calculate the mean and standard error.

Immunoprecipitation and western blotting. We incubated cell lysates with a Bop monoclonal or FLAG M2 (Sigma) antibody for $1 \mathrm{~h}$ at $4{ }^{\circ} \mathrm{C}$, followed by incubation for $1 \mathrm{~h}$ with protein-A sepharose beads (Sigma). After extensive washing, the precipitated proteins were analyzed by SDS-PAGE and transferred to nitrocellulose. Membranes were probed with the FLAG M2 or Bop monoclonal antibody. Western blots were developed with the enhanced chemiluminescence analysis system (Amersham).

Targeted deletion of Bop. We designed a construct to replace two exons encoding the putative zinc fingers and an adjacent domain (labeled exons 2 and 3 , respectively) with an oppositely directed $n e o^{\mathrm{r}}$ gene (Fig. 3a). Genomic fragments of $4.5 \mathrm{~kb}$ and $0.5 \mathrm{~kb}$ located upstream of exon 2 and downstream of exon 3, respectively, were cloned into the OSDUPDEL 1 vector 
(gift from N. Maeda and O. Smithies). Transfected SM1 embryonic stem cells were selected with G418 and gancyclovir, and correctly targeted integrations were identified by Southern hybridization. Correct integration of the short arm was indicated by a 2.2-kb $X m n \mathrm{I} / K p n \mathrm{I}$ fragment that hybridized with both probe 1 and a $n e o^{\mathrm{r}}$ probe, and a 2.8 -kb wildtype $X m n \mathrm{I} / \mathrm{X} m n \mathrm{I}$ fragment that hybridized only with probe 1 . Correct integration of the long arm was indicated by the presence of a $K p n \mathrm{I} / \mathrm{KpnI}$ fragment of 9-10 kb that hybridized with probe 2 , and the absence of a $4.5-\mathrm{kb} K p n \mathrm{I} / K p n \mathrm{I}$ fragment that could result only from the continued presence of the vector multicloning site. We carried out PCR genotyping of progeny; primer sequences are available upon request. We used RNA from $B o p^{-1-}$ or wildtype hearts at E9.0 for RT-PCR, using primers designed to amplify exons 1-4.

We derived four correctly targeted embryonic stem cell lines, two of which were injected into C57BL/6 blastocysts at E3.5 and transferred to (B6/D2) $F_{1}$ pseudopregnant females. We obtained two founders that passed the targeted allele to their progeny through the germ line.

Histochemistry. Wildtype and $B o p^{-/-}$embryos were collected at E9.25, fixed in $4 \%$ paraformaldehyde and processed for cryostat sectioning. The frozen sections were washed twice in PBS for 5 min each and once in $10 \%$ $\mathrm{H}_{2} \mathrm{O}_{2}$ for $5 \mathrm{~min}$, followed by two 5 -min washes with water and one 5 -min wash with PBS. The sections were incubated for $1 \mathrm{~h}$ with $8 \mu \mathrm{g} \mathrm{m}^{-1}$ of b-PG (biotinylated fragments of proteoglycan) ${ }^{38}$ in $10 \%$ calf serum/PBS and washed five times in PBS for 1 min each. We added a 1:500 dilution of horseradish peroxidase-conjugated streptavidin in $10 \%$ calf serum/PBS to the sections and incubated for $15 \mathrm{~min}$, followed by five 1-min washes in PBS. One tablet of DAKO DAB chromagen was dissolved in $10 \mathrm{ml} 0.05 \mathrm{M}$ Tris, $\mathrm{pH}$ 7.6. We applied the DAB chromagen to the sections twice for 5 min each, followed by two 5 -min washes in water.

TUNEL assay. We carried out TUNEL on transverse sections of four $B o p^{-l-}$ or wildtype embryos at E9.0 at the level of the outflow tract, as previously described ${ }^{33}$.

GenBank accession numbers. Mouse m-Bop1, U76373; mouse m-Bop2, U76374; chick m-Bop1, AA631880; chick m-Bop2, AA631881.

\section{Acknowledgments}

We are grateful to K. Artzt for help and advice; members of the Molecular Pathology Core (C. Pomajzl, J. Stark, J. Shelton and J. Richardson) for assistance with section in situ hybridizations; B. Tyson, D. Motola and C. Yamagishi for technical assistance; S. Johnson for preparation of graphics; C.B. Underhill for biotinylated fragments of proteoglycan and B.G. Bruneau for Irx 4 and Tbx5 plasmids. This work was supported by grants from the National Institute of Allergy and Infectious Diseases and the Texas Higher Education Coordinating Board (to P.D.G.), from the National Heart, Lung, and Blood Institute, National Institutes of Health and the D.W. Reynolds Cardiovascular Research Center (to E.N.O. and D.S.) and from March of Dimes and Smile Train Inc. (to D.S.). S.A.P. was supported by a training grant from the NIH.

\section{Competing interests statement}

The authors declare that they have no competing financial interests.

\section{Received 28 November 2001; accepted 5 March 2002}

1. Srivastava, D. \& Olson, E.N. A genetic blueprint for cardiac development. Nature $407,221-226(2000)$

2. Fishman, M.C. \& Chien, K.R. Fashioning the vertebrate heart: earliest embryonic decisions. Development 124, 2099-2117 (1997).

3. Hurle, J.M., Icardo, J.M. \& Ojeda, J.L. Compositional and structural heterogenicity of the cardiac jelly of the chick embryo tubular heart: a TEM, SEM and histochemical study. J. Embryol. Exp. Morphol. 56, 211-223 (1980)

4. Firulli, A.B. \& Olson, E.N. Modular regulation of muscle gene transcription: mechanism for muscle cell diversity. Trends Genet. 13, 364-369 (1997).

5. Srivastava, D. et al. Regulation of cardiac mesodermal and neural crest development by the bHLH transcription factor, dHAND. Nature Genet. 16, 154-160 (1997).

6. Biben, C. \& Harvey, R.P. Homeodomain factor Nkx2-5 controls left/right asymmetric expression of bHLH gene eHand during murine heart development. Genes Dev. 11, 1357-1369 (1997).

7. Pereira, F.A., Qiu, Y., Zhou, G., Tsai, M.J. \& Tsai, S.Y. The orphan nuclear receptor COUP-TFII is required for angiogenesis and heart development. Genes Dev. 13, 1037-1049 (1999).

8. Bao, Z.Z., Bruneau, B.G., Seidman, J.G., Seidman, C.E. \& Cepko, C.L. Regulation of chamber-specific gene expression in the developing heart by Irx4. Science 283, 1161-1164 (1999).

9. Nakagawa, O., Nakagawa, M., Richardson, J.A., Olson, E.N. \& Srivastava, D. HRT1, HRT2, and HRT3: a new subclass of bHLH transcription factors marking specific cardiac, somitic, and pharyngeal arch segments. Dev. Biol. 216, 72-84 (1999).

10. Cheung, P., Allis, C.D. \& Sassone-Corsi, P. Signaling to chromatin through histone modifications. Cell 103, 263-271 (2000).

11. Marmorstein, R. \& Roth, S.Y. Histone acetyltransferases: function, structure, and catalysis. Curr. Opin. Genet. Dev. 11, 155-161 (2001).

12. Khochbin, S., Verdel, A., Lemercier, C. \& Seigneurin-Berny, D. Functional significance of histone deacetylase diversity. Curr. Opin. Genet. Dev. 11, 162-166 (2001).

13. Rea, $\mathrm{S}$. et al. Regulation of chromatin structure by site-specific histone $\mathrm{H} 3$ methyltransferases. Nature 406, 593-599 (2000).

14. Nakayama, J., Rice, J.C., Strahl, B.D., Allis, C.D. \& Grewal, S.I. Role of histone H3 lysine 9 methylation in epigenetic control of heterochromatin assembly. Science 292, 110-113 (2001).

15. Jenuwein, T. \& Allis, D. Translating the histone code. Science 293, 1074-1080 (2001).

16. Lutterbach, B. et al. ETO, a target of $t(8 ; 21)$ in acute leukemia, interacts with the $\mathrm{N}-\mathrm{CoR}$ and $\mathrm{mSin} 3$ corepressors. Mol. Cell Biol. 18, 7176-7184 (1998).

17. Gelmetti V. et al. Aberrant recruitment of the nuclear receptor corepressorhistone deacetylase complex by the acute myeloid leukemia fusion partner ETO. Mol. Cell Biol. 18, 7185-7191 (1998).

18. Hwang, I. \& Gottlieb, P.D. Bop: a new T-cell-restricted gene located upstream of and opposite to mouse CD8b. Immunogenetics 42, 353-361 (1995).

19. Hwang, I. \& Gottlieb, P.D. The Bop gene adjacent to the mouse CD8b gene encodes distinct zinc- finger proteins expressed in CTLs and in muscle. J. Immunol. 158, 1165-1174 (1997).

20. Hamburger, V. \& Hamilton, H. A series of normal stages in the development of the chick embryo. J. Morphol. 88, 49-82 (1951).

21. Lints, T.J., Parsons, L.M., Hartley, L., Lyons, I. \& Harvey, R.P. Nkx-2.5: a novel murine homeobox gene expressed in early heart progenitor cells and their myogenic descendants. Development 119, 419-431 (1993).

22. Komuro, I. \& Izumo, S. Csx: a murine homeobox-containing gene specifically expressed in the developing heart. Proc. Natl Acad. Sci. USA 90, 8145-8149 (1993).

23. Hollenberg, S.M., Sternglanz, R., Cheng, P.F. \& Weintraub, H. Identification of a new family of tissue-specific basic helix-loop-helix proteins with a two-hybrid system. Mol. Cell Biol. 15, 3813-3822 (1995).

24. Lutterbach, B., Sun, D., Schuetz, J. \& Hiebert, S.W. The MYND motif is required for repression of basal transcription from the multidrug resistance 1 promoter by the $\mathrm{t}(8 ; 21)$ fusion protein. Mol. Cell Biol. 18, 3604-3611 (1998).

25. Yoshida, M., Horinouchi, S. \& Beppu, T. Trichostatin A and trapoxin: novel chemical probes for the role of histone acetylation in chromatin structure and function. Bioessays 17, 423-430 (1995).

26. Camenisch, T.D. et al. Disruption of hyaluronan synthase-2 abrogates normal cardiac morphogenesis and hyaluronan-mediated transformation of epithelium to mesenchyme. J. Clin. Invest. 106, 349-360 (2000).

27. Bernanke, D.H. \& Orkin, R.W. Hyaluronate binding and degradation by cultured embryonic chick cardiac cushion and myocardial cells. Dev. Biol. 106, 360-367 (1984).

28. O'Brien, T.X., Lee, K.J. \& Chien, K.R. Positional specification of ventricular myosin light chain 2 expression in the primitive murine heart tube. Proc. Natl Acad. Sci. USA 90, 5157-5161 (1993).

29. Kubalak, S.W., Miller-Hance, W.C., O'Brien, T.X., Dyson, E. \& Chien, K.R. Chamber specification of atrial myosin light chain-2 expression precedes septation during murine cardiogenesis. J. Biol. Chem. 269, 16961-16970 (1994).

30. Srivastava, D., Cserjesi, P. \& Olson, E.N. A subclass of bHLH proteins required for cardiac morphogenesis. Science 270, 1995-1999 (1995).

31. Bruneau, B.G. et al. Cardiac expression of the ventricle-specific homeobox gene Irx4 is modulated by Nkx2-5 and dHAND. Dev. Biol. 217, 266-277 (2000).

32. Bruneau, B.G. et al. Chamber-specific cardiac expression of $\mathrm{Tb} \times 5$ and heart defects in Holt-Oram syndrome. Dev. Biol. 211, 100-108 (1999).

33. Yamagishi, $\mathrm{H}$. et al. The combinatorial activities of $\mathrm{Nkx2.5}$ and dHAND are essential for cardiac ventricle formation. Dev. Biol. 239, 190-203 (2001).

34. McFadden, D.G. et al. A GATA-dependent right ventricular enhancer controls dHAND transcription in the developing heart. Development 127, 5331-5341 (2000).

35. McKinsey, T.A., Zhang, C.L., Lu, J. \& Olson, E.N. Signal-dependent nuclear export of a histone deacetylase regulates muscle differentiation. Nature 408, 106-111 (2000).

36. Jenuwein, T. Re-SET-ting heterochromatin by histone methyltransferases. Trends Cell Biol. 11, 266-273 (2001).

37. Yamagishi, H., Garg, V., Matsuoka, R., Thomas, T. \& Srivastava, D. A molecular pathway revealing a genetic basis for human cardiac and craniofacial defects. Science 283, 1158-1161 (1999).

38. Green, S.J., Tarone, G. \& Underhill, C.B. Distribution of hyaluronate and hyaluronate receptors in the adult lung. J. Cell Sci. 90, 145-156 (1988). 